\section{RÉPLICA DE LA ESTRATEGIA SANITARIA NACIONAL DE PREVENCIÓN Y CONTROL DE LA TUBERCULOSIS}

\section{[REPLY OF THE PERUVIAN NATIONAL HEALTH STRATEGY FOR TUBERCULOSIS PREVENTION AND CONTROL]}

\author{
Oswaldo Jave ${ }^{1, a, b}$, Félix Llanos-Tejada1,b
}

Sr. Editor. Leemos con agradola información y opinión del Sr. Julio Maquera-Afaray, estudiante de Medicina de Tacna, la que como representantes de la Estrategia Sanitaria Nacional de Prevención y Control de la Tuberculosis (ESNPyCTB) del Ministerio de Salud (MINSA) compartimos.

Es sabido que los niños representan la población con mayor riesgo de progresar a enfermedad por tuberculosis (TB) luego de una infección, incluyendo mayor riesgo de presentar formas graves de enfermedad y complicaciones $^{(1)}$, por lo que en nuestro medio la quimioprofilaxis con isoniacida ha sido orientada a esta población en particular, lo que equivale a tratamiento preventivo. En los años 1980-1990 los casos de meningoencefalitis TB infantil eran frecuentes, dejando como secuelas hidrocefalia permanente y trastornos neurológicos ${ }^{(2)}$. La literatura médica menciona la necesidad de proteger preventivamente también a los diabéticos y, en décadas recientes, se extendió a los casos de personas con VIH-SIDA por la alta tasa de morbi-mortalidad por TB, por lo tanto, tributario de tratamiento con quimioprofilaxis con isoniazida en el Perú y el mundo ${ }^{(1-4)}$.

Con el advenimiento de nuevos métodos diagnósticos de infección TB, se han venido realizando estudios recientes que buscan evaluar el impacto del tratamiento de la TB latente en población adulta, especialmente, en población en riesgo ${ }^{(4-6)}$. Estos estudios han podido determinar el impacto positivo en el ámbito de la salud pública de esta actividad preventiva.

Es así que coincidimos en la necesidad de ampliar la cobertura de la quimioprofilaxis a otros grupos poblacionales de alto riesgo de enfermedad TB, como son los diabéticos, pacientes con insuficiencia renal, neumoconiosis, personal de salud recientemente infectado, usuarios de corticoides e inmuno-moduladores (enfermos

\footnotetext{
Estrategia Sanitaria Nacional de Prevención y Control de la

Tuberculosis, Ministerio de Salud. Lima, Perú.

a Responsable de la ESNPyCT; ${ }^{\text {b }}$ Médico neumólogo.
}

Recibido: 23-05-10 Aprobado: 02-06-10 respiratorios crónicos, enfermos del tejido conectivo, etc.), transplantados, entre otros grupos focalizados.

La norma técnica de salud para el control de la tuberculosis (2), actualmente vigente, en el subtítulo "Estudio de contactos y quimioprofilaxis" (página 97) menciona lo siguiente en indicaciones:

Contactos de personas con tuberculosis pulmonar en situaciones clínicas especiales, en los que podrá emplearse quimioprofilaxis únicamente por indicación médica, cuando presenten: diabetes, silicosis, gastrectomía, enfermedades hematológicas malignas, tratamiento con inmunosupresores, insuficiencia renal terminal y transplantes renales, terapias prolongadas con corticoides y enfermedades caquectizantes.

Esperamos que los usuarios de la norma de TB puedan encontrar, previa revisión, información apropiada.

\section{Conflictos de interés}

Los autores son miembros de la ESNPyCTB.

\section{REFERENCIAS BIBLIOGRÁFICAS}

1. World Health Organization. Treatment of tuberculosis. Guidelines. 4th ed. Geneva: WHO; 2010.

2. Perú, Ministerio de Salud. Norma técnica de salud para el control de la tuberculosis. Lima: MINSA/DGSP; 2006.

3. Gonzáles-Martín J, García-García JM, Anibarro L, Vidal R, Esteban J, Blanquer R, et al. Documento de consenso sobre diagnóstico, tratamiento y prevención de la tuberculosis. Arch Bronconeumol 2010; 46(5): 255-74.

4. Mack U, Migliori GB, Sester M, Rieder HL, Ehlers S, Goletti D, et al. Consensus Statement. LTBI: latent tuberculosis infection or lasting immune responses to $M$. tuberculosis? A TBNET Consensus Statement. Eur Respir J. 2009; 33(5): 956-73.

5. Cailleaux-Cezar M, de A Melo D, Xavier GM, de Salles CL, de Mello FC, Ruffino-Netto A, el al. Tuberculosis incidence among contacts of active pulmonary tuberculosis. Int J Tuberc Lung Dis. 2009; 13(2): 190-95.

6. Holland DP, Sanders GD, Hamilton CD, Stout JE. Costs and cost-effectiveness of four treatment regimens for latent tuberculosis infection. Am J Respir Crit Care Med. 2009; 179(11): 1055-60.
Correspondencia: Oswaldo Jave Castillo Dirección: Av. Salaverry cuadra $8 \mathrm{~s} / \mathrm{n}$, Lima11, Perú. Correo electrónico: rigeljave2008@yahoo.es 